\title{
Effects of Sitagliptin as Monotherapy and Add-On to Metformin on Weight Loss among Overweight and Obese Patients with Type 2 Diabetes: A Systematic Review and Meta-Analysis
}

\author{
Authors \\ Leila Janani ${ }^{1}$, Hadi Bamehr ${ }^{2}$, Kiarash Tanha ${ }^{1}$, Parastoo Mirzabeigi ${ }^{3}$, Hamed Montazeri ${ }^{4}$, Parastoo Tarighi ${ }^{5}$
}

\section{Affiliations}

1 Department of Biostatistics, School of Public Health, Iran University of Medical Sciences, Tehran, Iran

2 Department of Medical Biotechnology, Biotechnology Research Center, Pasteur Institute of Iran, Tehran, Iran

3 Department of Clinical Pharmacy, School of Pharmacy, Iran University of Medical Sciences, Tehran, Iran

4 Department of Pharmacognosy and Pharmaceutical Biotechnology, School of Pharmacy, Iran University of Medical Sciences, Tehran, Iran

5 Department of Medical Biotechnology, Faculty of Allied Medicine, Iran University of Medical Sciences, Tehran, Iran

Key words

Systematic review, Meta-analysis, Randomised clinical trial, Type 2 diabetes, Sitagliptin, Overweight, Metformin

received 19.02 .2021

revised 18.06 .2021

accepted 18.07.2021

published online 13.08 .2021

Bibliography

Drug Res 2021; 71: 477-488

DOI 10.1055/a-1555-2797

ISSN 2194-9379

(C) 2021. Thieme. All rights reserved.

Georg Thieme Verlag, Rüdigerstraße 14,

70469 Stuttgart, Germany

Correspondence

Parastoo Tarighi

Iran University of Medical Sciences - Medical Biotechnology

Shahid Hemmat Highway next to Milad Tower,

Tehran,

1449614535

Iran (the Islamic Republic of)

Tel.: 00982186704715, Fax: 00982188622533

tarighi.p@iums.ac.ir

\section{ABSTRACT}

Background Sitagliptin is known as an antidiabetic agent inhibiting the dipeptidyl peptidase-4. Although sitagliptin may influence weight, controversial results have been reported, and there is no general agreement on this issue. Therefore, this study assessed the effect of sitagliptin as monotherapy and add-on therapy to metformin on weight reduction in overweight or obese cases with type 2 diabetes.

Methods We reviewed the following databases to identify all relevant papers published until 1st April 2021: Web of Science, MEDLINE, Embase, Scopus, Cochrane Central Register of Controlled Trials Cochrane Library, and Google Scholar. The research included all clinical trials investigating the effect of sitagliptin in obese or overweight adult patients with type 2 diabetes without any language restriction.

Results In total, eighteen randomized controlled trials with 2009 participants were included in our meta-analysis. Results showed supplementation of sitagliptin has led to weight loss for sitagliptin treated (MD -0.99; $95 \% \mathrm{Cl} ;(-1.87,-0.12)$; $\mathrm{p}=0.026))$ and sitagliptin + metformin treated groups (MD -1.09; $95 \% \mathrm{Cl} ;(-1.69,-0.49) ; \mathrm{p}<0.001)$ ). Also, the intervention has influenced body mass index in sitagliptin treated (MD -0.23; $95 \% \mathrm{Cl} ;(-0.45,0.02) ; \mathrm{p}=0.033)$ ) and sitagliptin + metformin treated groups (MD $-0.52 ; 95 \% \mathrm{Cl} ;(-0.96$, $0.08) ; p=0.020)$ ) comparing to placebo.

Conclusion Our results demonstrated that sitagliptin administration with or without metformin might reduce the body weight and body mass index if these drugs are taken for more than 6 months. 


\section{ABBREVIATIONS \\ BMI Body Mass Index \\ CENTRAL Cochrane Central Register of Controlled Trials \\ DPP-4 Dipeptidyl Peptidase-4 \\ FPG Fasting Plasma Glucose \\ GLP-1 Glucagon-Like Peptide-1 \\ HbA1c Glycated Hemoglobin \\ PRISMA Preferred Reporting Items for the Systematic \\ Reviews and Meta-Analyses \\ PCOS Polycystic Ovary Syndrome \\ PPG two-hour Postprandial Glucose \\ WHO World Health Organization \\ RCTs Randomized Controlled Trials \\ PBO Placebo \\ MET Metformin \\ SIT Sitagliptin}

\section{Introduction}

World Health Organization (WHO) explains obesity as an abnormal fat accumulation or excess body adiposity and a relapsing disease [1]. Obesity and overweight issues have attracted global attention due to their increasing prevalence, estimated at $38 \%$ of the world population in 2038 [2]. In recent years, obesity has increased pri- marily due to urbanization, reduced physical activity, and increased availability of food supplies [3]. Furthermore, the economic effect of obesity leads to the simultaneous expansion of social, medical, and public health costs [4]. There is a significant association between obesity and many chronic diseases, particularly type 2 diabetes [5]. Obesity or weight gain is associated with an increased risk of type 2 diabetes. Besides, obesity is a risk factor strongly related to the increased risk of cardiovascular disease [6, 7], responsible for around 70 to $80 \%$ of type 2 diabetes-related mortality $[8,9]$.

It is well established that weight reduction is associated with a low risk of developing type 2 diabetes $[10,11]$. The weight loss issue has received considerable critical attention in managing type 2 diabetes, but weight loss strategies suffer from limited success [12]. Weight loss leads to improved glycemic control and a decreased need for glucose-lowering drugs in patients with diabetes. In overweight or obese type 2 diabetic adults, dietary recommendations, physical activity, and behavioral therapy are strongly considered to achieve weight loss. Based on the strong evidence, obesity management has a valuable role in slowing the progression of diabetes. In this regard, choosing the antidiabetic medications associated with weight loss in type 2 diabetic patients who have overweight or obese can be beneficial [13]. So far, a great deal of attention has been paid to the pharmacological approach. Antidiabetic agents such as insulin, sulfonylureas, and thiazolidinediones cause weight gain as their side-effects $[14,15]$. Sitagliptin has been shown to preserve $\beta$-cell function and improve glycated hemoglobin (HbA1c), 2-h postprandial glucose

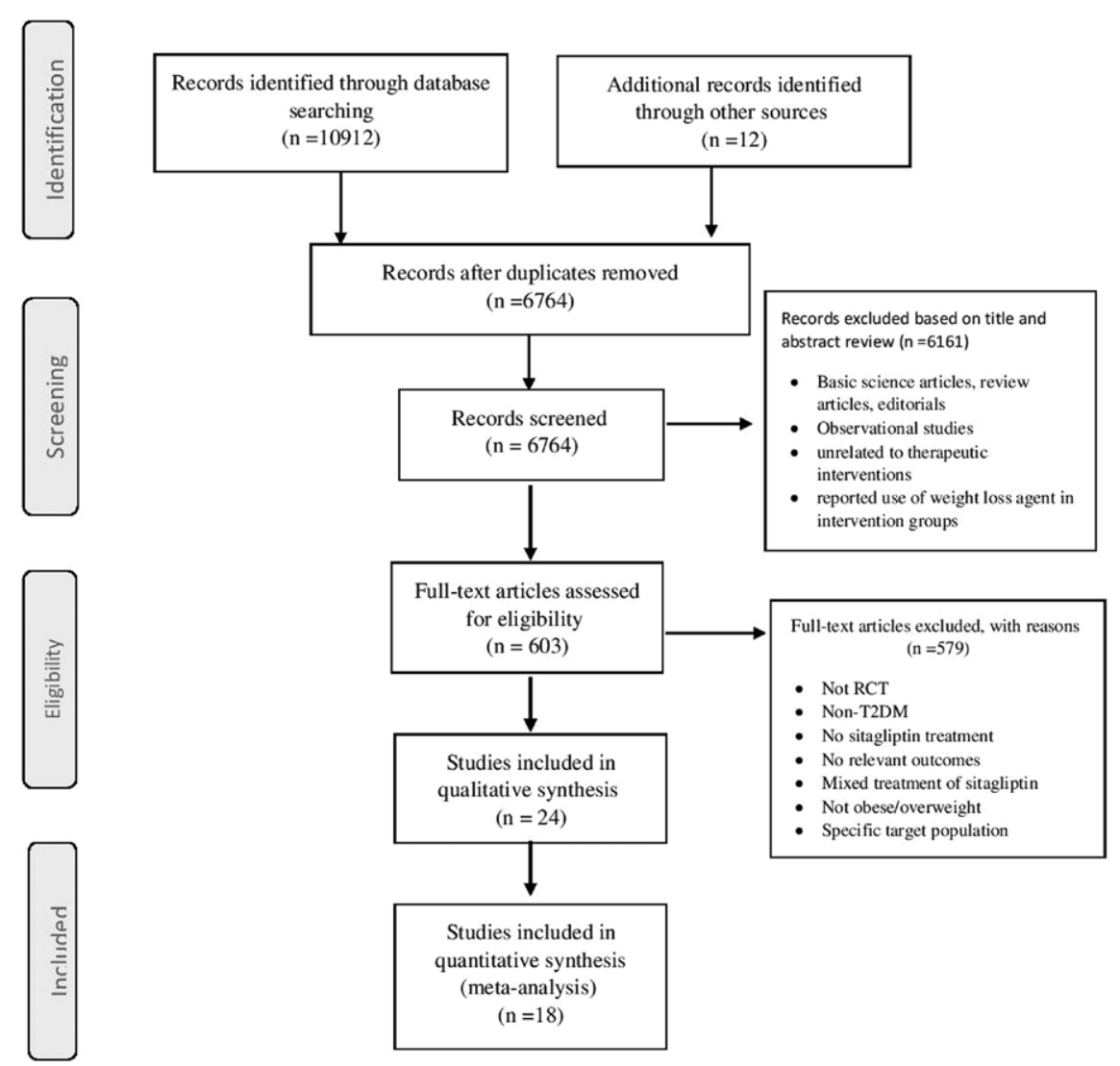

- Fig. 1 PRISMA flow diagram. 


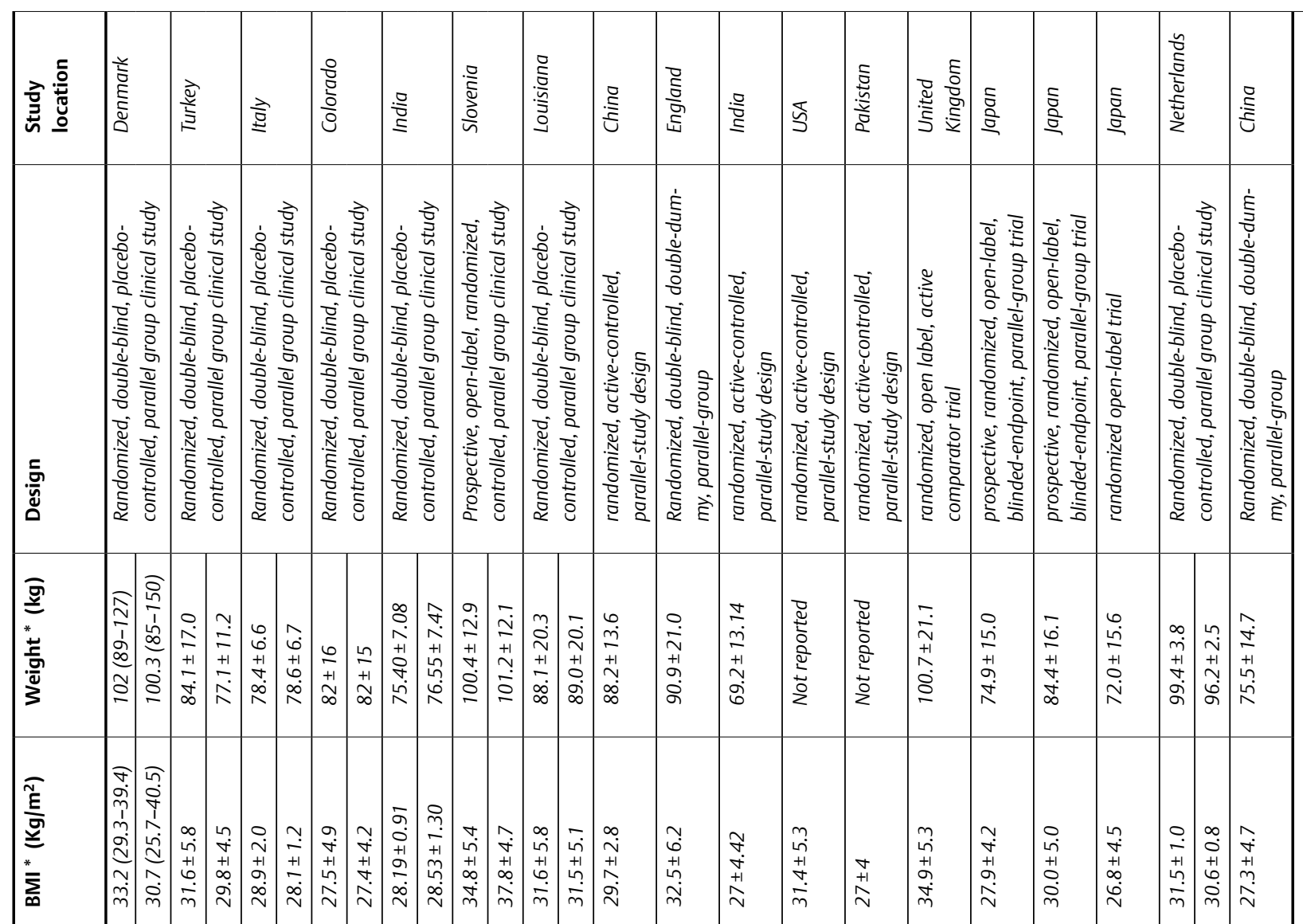

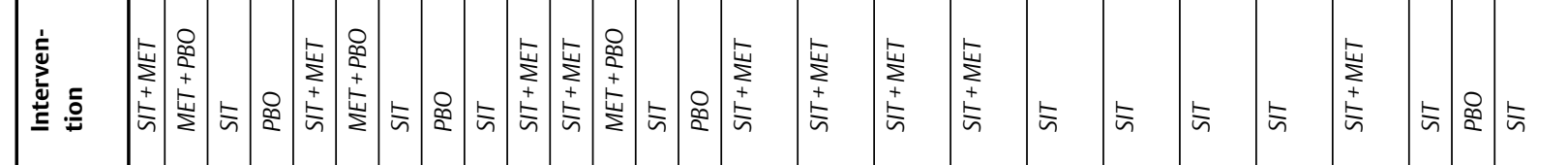

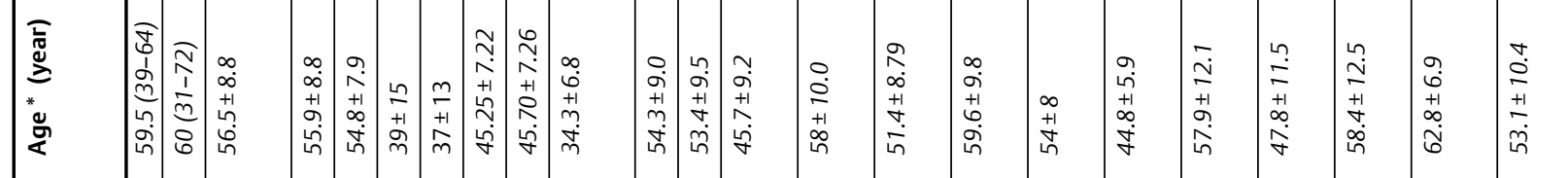

鿷事

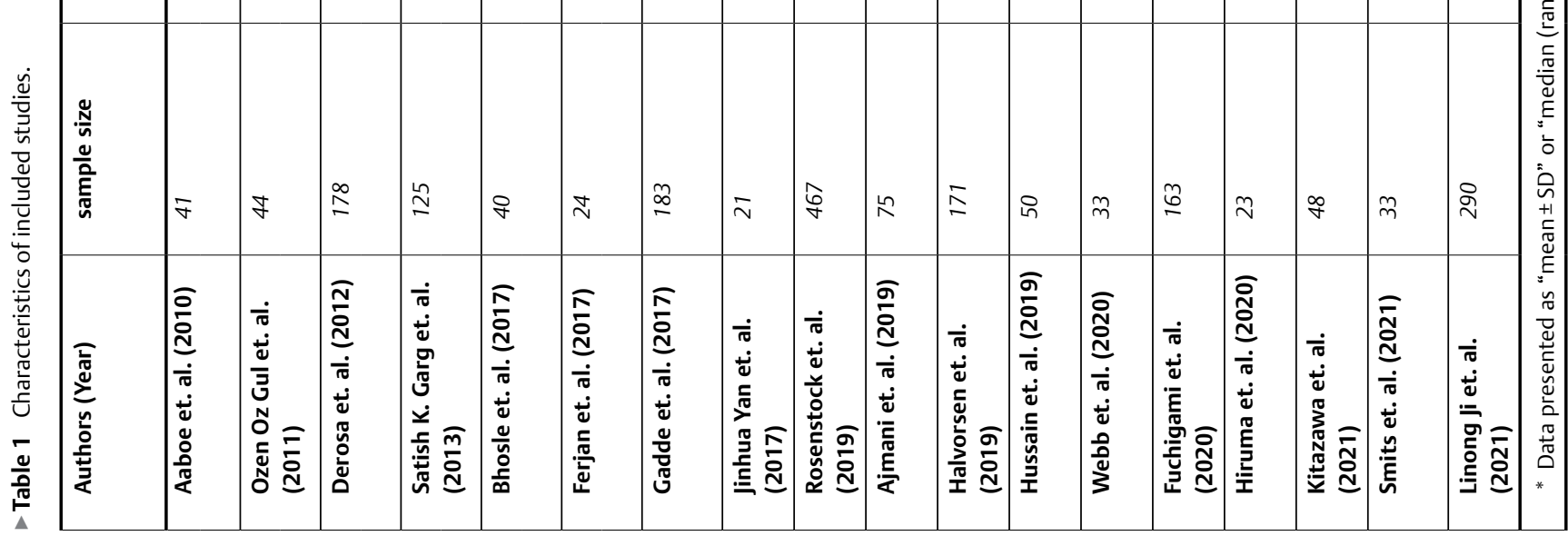


a

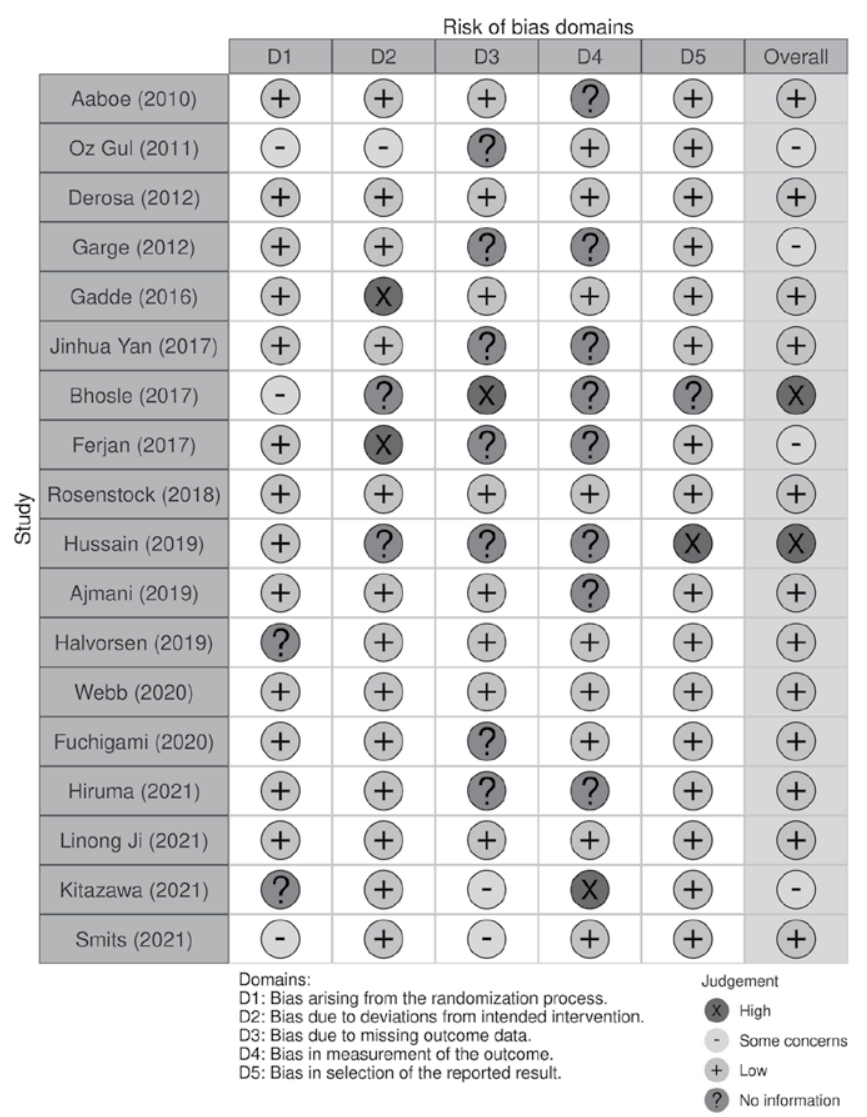

b

Bias arising from the randomization process Bias due to deviations from intended interventions Bias due to missing outcome data Bias in measurement of the outcome Bias in selection of the reported result Overall risk of bias

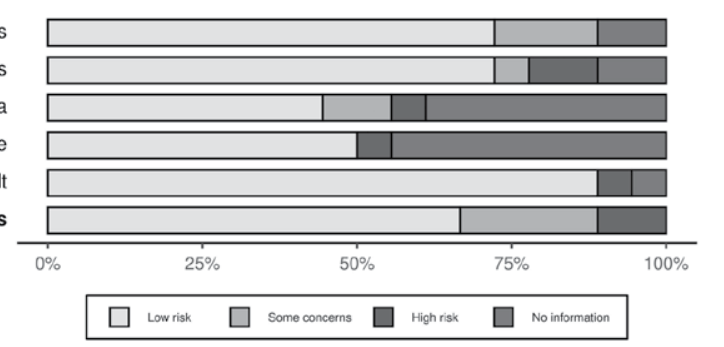

- Fig. 2 a. Cochrane risk-of-bias tool for randomized trials (individual studies). b. Cochrane risk-of-bias tool for randomized trials (overall bias in domains).

(PPG), fasting plasma glucose (FPG), and hyperglycemia in individuals with type 2 diabetes [16, 17]. As the first oral dipeptidyl peptidase-4 (DPP-4) inhibitor, sitagliptin or gliptin was approved by the United States Food and Drug Administration (FDA) in 2006. It inhibits the DPP-4 receptor [18]. DPP-4 stimulates glucagon release and decreases insulin secretion by inactivating incretin such as glucagonlike peptide-1 (GLP-1) [19]. A severe reduction or loss of the incretin effect has also been reported in patients with type 2 diabetes [20]. GLP-1 is an essential incretin hormone promoting proliferation/neogenesis ratio and inhibiting beta cells' apoptosis [21]. GLP-1 delays nutrient delivery by delaying gastric emptying, reducing postprandial glucose excursions [22], and might reinforce the change in eating behavior [23]. Accordingly, it is assumed that the enhancement of endogenous incretin induces weight loss through the inhibition of DPP-4. More recently, sitagliptin has been used as a treatment option for metformin failure in diabetes, polycystic ovary syndrome (PCOS), and as a preventive agent against the progression of type 2 diabetes and its comorbidities [24, 25]. Tran et al. conducted a meta-analysis about the efficacy of DPP-4 inhibitor compared to GLP-1 in managing type 2 diabetes. They concluded that GLP-1 performs better in weight loss and does not influence sitagliptin [26]. Juan's meta-analysis about DDP-4 inhibitors failed to address the efficacy of sitagliptin on weight loss [27]. There are also discrepancies and sometimes controversies between clinical studies investigating the effect of sitagliptin on weight loss [28-32]. Thus, the present systematic review and meta-analysis are designed to assess the impact of sitagliptin on weight loss in type 2 diabetic obese or overweight adults. 


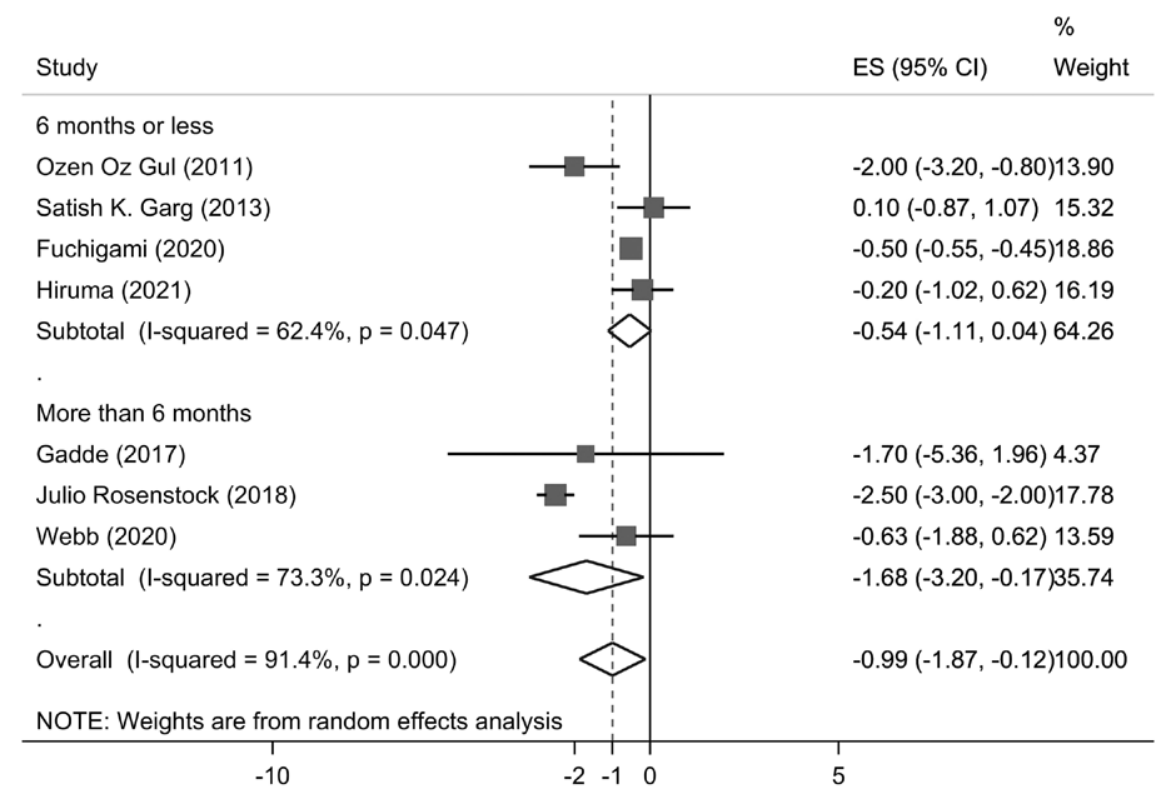

- Fig. 3 Point estimation of mean differences with $95 \% \mathrm{Cl}$ for body weight in single drug groups for before-after intervention. Forest plot for beforeafter comparison of body weight means in people who received sitagliptin as a monotherapy using random-effect-model meta-analysis. (ES; effect size, Weight: study weight in meta-analysis).

\begin{tabular}{|c|c|c|}
\hline Study & $\mathrm{ES}(95 \% \mathrm{Cl})$ & $\begin{array}{l}\% \\
\text { Weight }\end{array}$ \\
\hline \multicolumn{3}{|l|}{6 months or less } \\
\hline Derosa (2012) & $-1.00(-2.33,0.33)$ & 7.69 \\
\hline Ferjan (2017) & $1.00(-0.26,2.26)$ & 8.01 \\
\hline K. Aaboe (2010) & $-2.15(-24.87,20.57)$ & 0.07 \\
\hline Smits (2021) & $-1.00(-1.98,-0.02)$ & 9.32 \\
\hline Halvorsen (2019) & $-0.81(-0.85,-0.77)$ & 12.40 \\
\hline Subtotal $(I-$ squared $=51.3 \%, p=0.084)$ & $-0.58(-1.23,0.07)$ & 37.48 \\
\hline \multicolumn{3}{|l|}{. } \\
\hline \multicolumn{3}{|l|}{ More than 6 months } \\
\hline Derosa (2012) & $-2.50(-3.76,-1.24)$ & 7.99 \\
\hline Jinhua Yan (2017) & $-1.70(-2.76,-0.64)$ & 8.94 \\
\hline Ajmani (2019) & $-0.23(-0.58,0.12)$ & 11.89 \\
\hline Ajmani (2019) & $-0.36(-0.83,0.11)$ & 11.53 \\
\hline Kitazawa (2021) & $-0.50(-1.09,0.09)$ & 11.05 \\
\hline Linong Ji (2021) & $-3.79(-4.37,-3.21)$ & 11.12 \\
\hline Subtotal $(I-$ squared $=96.0 \%, p=0.000)$ & $-1.48(-2.69,-0.27)$ & 62.52 \\
\hline . & & \\
\hline Overall $(I-$ squared $=92.6 \%, p=0.000)$ & $-1.09(-1.69,-0.49)$ & 100.00 \\
\hline \multicolumn{3}{|l|}{ NOTE: Weights are from random effects analysi\$ } \\
\hline $\begin{array}{lll}1 & \\
-24.9 & 0\end{array}$ & & \\
\hline
\end{tabular}

Fig. 4 Point estimation of mean differences with $95 \% \mathrm{Cl}$ for body weight in multiple drug groups for before-after intervention. Forest plot for beforeafter comparison of body weight means in people who received sitagliptin + metformin using random-effect-model meta-analysis (ES; effect size, Weight: study weight in meta-analysis). 


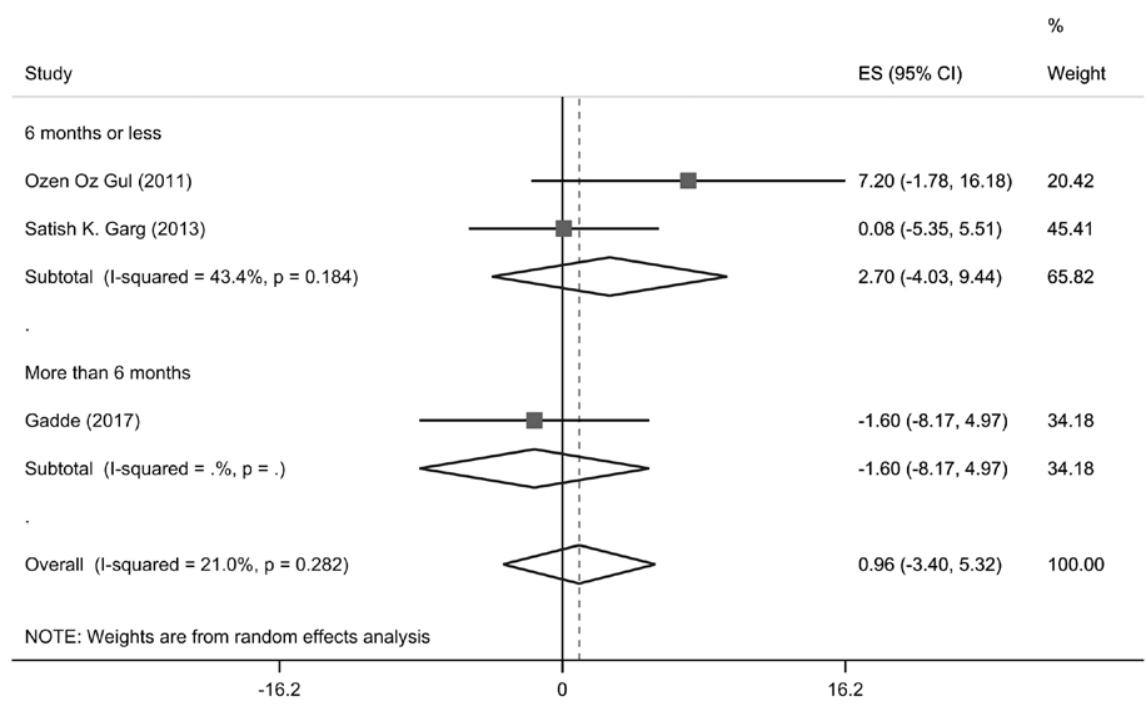

- Fig. 5 Point estimation of mean differences with $95 \% \mathrm{Cl}$ for body weight in single drug groups for comparison of sitagliptin group vs. placebo. Forest plot using random-effect-model meta-analysis for comparison of body weight means in people who received sitagliptin as monotherapy vs. people who received placebo group (ES; effect size, Weight: study weight in meta-analysis).

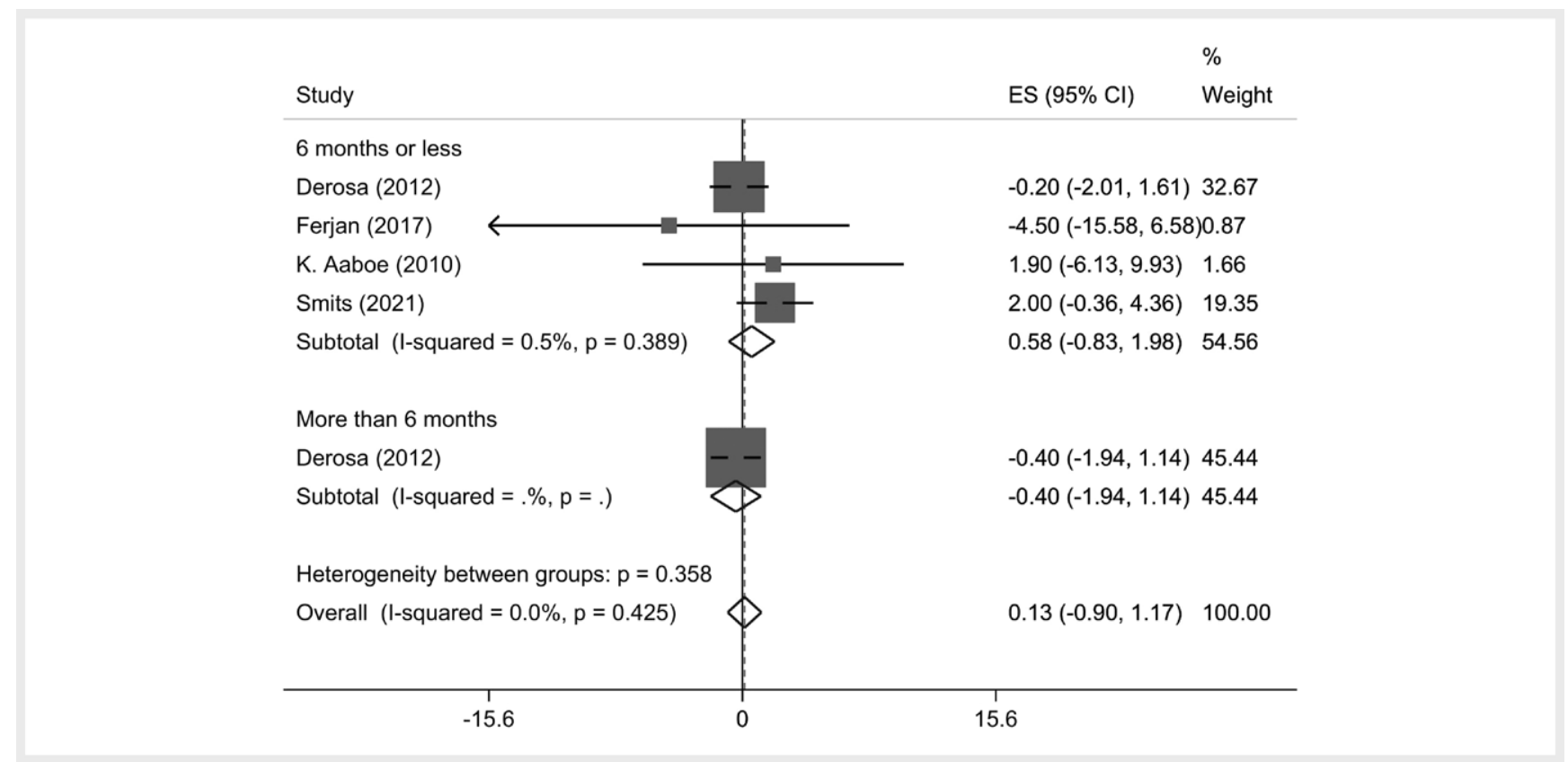

- Fig. 6 Point estimation of mean differences with $95 \% \mathrm{Cl}$ for body weight in multiple drug groups for comparison of sitagliptin group vs. placebo. Forest plot using fixed-effect-model meta-analysis for comparison of body weight means in people who received sitagliptin + metformin vs. people who received placebo (ES; effect size, Weight: study weight in meta-analysis).

\section{Material \& Methods}

The Preferred Reporting Items for Systematic Reviews and MetaAnalysis (PRISMA) guideline was used to report the review.

\section{Literature Search}

We searched the following databases to find related Clinical Trials published until 1st April 2021: Cochrane Central Register of Con- trolled Trials (CENTRAL), MEDLINE [PubMed], Scopus, Embase, and Web of Science. In addition, we searched the databases of the International Standard Randomized Controlled Trial Number (ISRCTN) Registry and Meta-Register for references of Randomized Controlled Trials (RCTs) for ongoing relevant publications. The search syntax was contained related keywords as follow: ((Weight or Obesity * or BMI or "Body Mass" or Quetelet *) and (Sitagliptin 


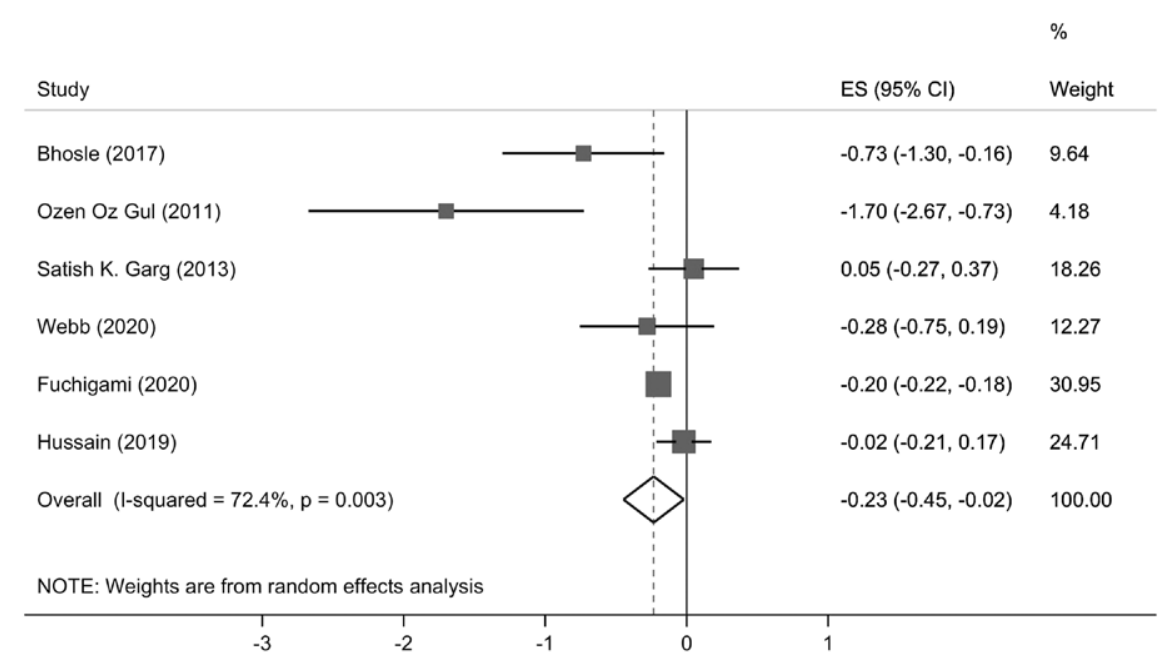

- Fig. 7 Point estimation of mean differences with $95 \% \mathrm{Cl}$ for BMI in single drug groups for before-after intervention. Forest plot for before-after comparison of BMI means in people who received sitagliptin as a monotherapy using random-effect-model meta-analysis (ES; effect size, Weight: study weight in meta-analysis).

or Januvia or Antiobesity* or (MK and 0431) or (Prader - Willi and Syndrome))). We restricted the search to the studies conducted on humans. However, the research also included the literature published in any language. Additionally, after finalizing the search strategy and defining the inclusion criteria, an expletive search was conducted by searching through related articles' references.

\section{Inclusion and Exclusion Criteria}

Eligible RCTs written in any language were only included in the analysis if they met the following framework: monotherapy with sitagliptin, combined treatment of sitagliptin with metformin, treatment duration of at least two months, providing weight and/or body mass index (BMI) values and the criteria for selecting the subjects were as follows: All the participants who were aged 18 years old and older at the beginning of the study with a medical history of type 2 diabetes and were classified as overweight or obese based on accepted standards such as BMI and body weight. Also, English abstracts of non-English papers were assessed if possible. In multiple publications of a study, the latest version with the most enrolled participants has been included. Studies written in other languages were translated sufficiently in detail if required.

\section{Study Selection and Eligible Papers}

Selected titles and abstracts were scanned by two reviewers independently (HB, KT) to identify eligible studies for further assessment. First, the abstracts were screened to remove irrelevant studies that did not meet inclusion criteria. Then, the inspection continued by reviewing the full text of all potentially relevant studies undertaken by two reviewers independently (HB, KT). Finally, according to the criteria, the study's final stage was performed to reach an agreement and select the final papers. Controversies were resolved by consulting with a third person (LJ).

We assessed the bias risk of included studies using the Cochrane tool for assessing the risk of bias for randomized trials (RoB 2 tool), which is a domain-based critical assessment of the following issues) randomization process; intended intervention, missing outcome data, measurement of the outcome, reported results; which answers lead to judgments of "low risk of bias," "some concerns," or "high risk of bias" [33]. First, the differences between results were surveyed, and then they were resolved through discussion.

\section{Data Extraction}

Two reviewers (Hadi Bamehr, Kiarash Tanha) separately extracted the data from all included studies. The disagreement between the reviewers was resolved by a discussion with the third author (LJ). Eligible studies were reviewed, and the following data were collected: first authors' name, publication year, study location, publication type, study design, number of the participants in the intervention and control groups, duration of the intervention, characteristics of the patients, age, sex, paramount inclusion and exclusion criteria, and outcomes including BMI, weight measures at baseline and end of each study.

\section{Statistical analysis}

Stata software version 13 (Stata Corp., College Station, TX, USA) was used for data analysis. Since all study outcomes ranged on a continuous scale, pooled estimates were reported as Mean Difference (MD) and 95\% Confidence Interval (CI). MDs were calculated for before-after comparison in the intervention group and for comparing weight and BMI in intervention groups compared to placebo at the end of the study. Due to a lack of relevant data, we could not compare changes from baseline in control and intervention groups. Heterogeneity assessment between the studies was performed using the 12 statistic (the 12 values of 25,50 , and $75 \%$ indicated low, moderate, and high heterogeneities, respectively), representing the percentage of the variability in effect estimates that is due to heterogeneity rather than sampling error [34]. Cochran's Q statistic was also used to determine the statistical significance of 


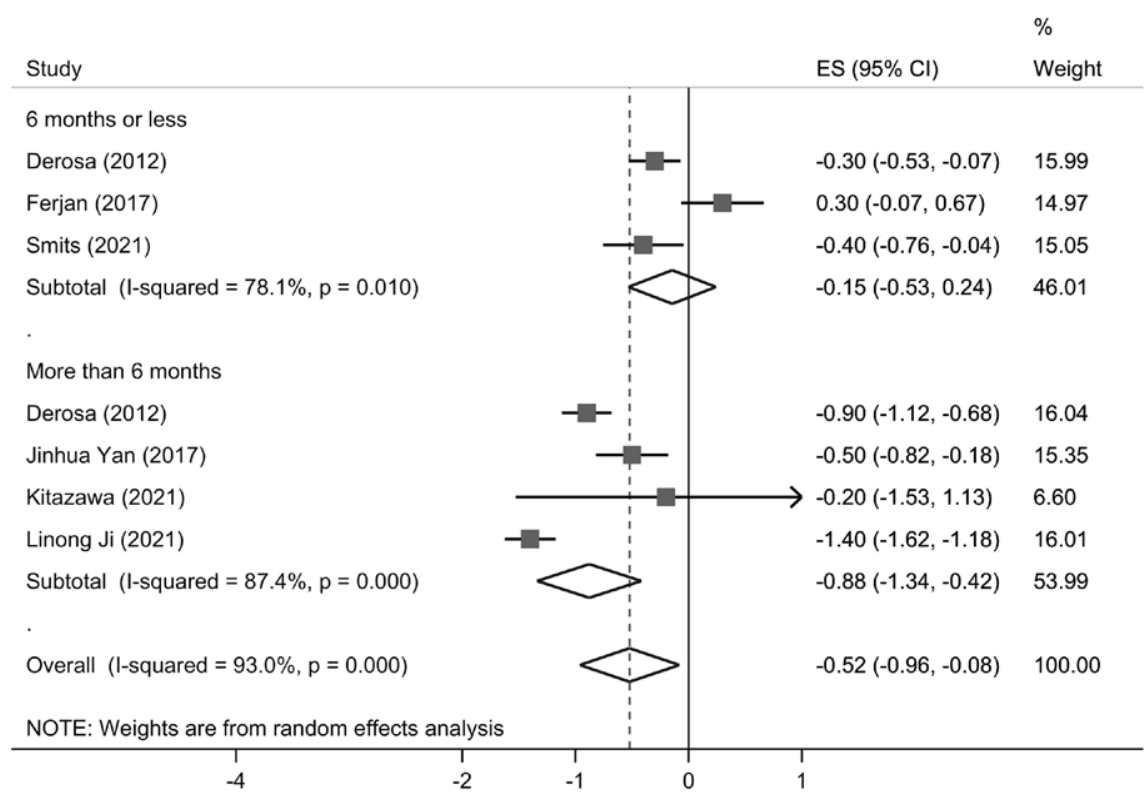

- Fig. 8 Point estimation of mean differences with $95 \% \mathrm{Cl}$ for BMI in Multiple drug groups for before-after intervention. Forest plot for before-after comparison of BMI means in people who received sitagliptin + metformin using random-effect-model meta-analysis (ES; effect size, Weight: study weight in meta-analysis).

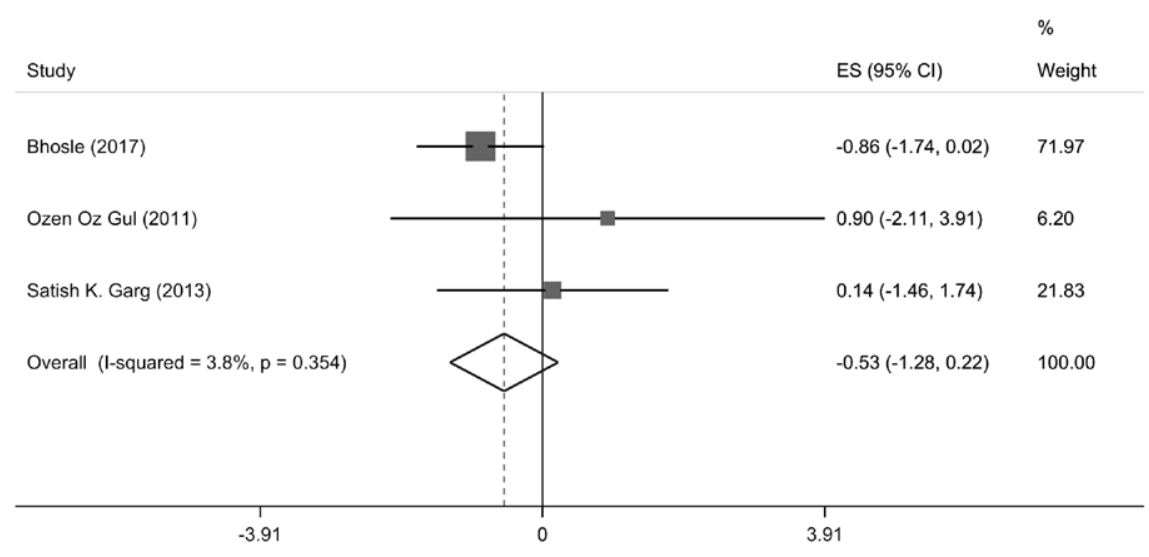

-Fig. 9 Point estimation of mean differences with $95 \% \mathrm{Cl}$ for BMI in single drug groups for comparison of sitagliptin group vs. placebo. Forest plot using fixed-effect-model meta-analysis for comparison of BMI means in people who received sitagliptin as monotherapy vs. people who received placebo group (ES; effect size, Weight: study weight in meta-analysis).

the heterogeneity. Also, subgroup analyses were carried out to determine whether the association differed depending on the type of treatment (single drug or multiple drugs) and supplementation duration (six months or less and more than six months). The Visual inspection of funnel plots assessed the publication bias. The publication bias was also evaluated in more than three studies using Egger's test for asymmetry of the funnel plots. A P-value of $<0.10$ was considered evidence of bias. All the statistical tests of the data were two-tailed, and a P-value less than 0.05 was statistically significant for all the tests except publication bias tests.

\section{Results}

A total of 10924 potential citations were identified in our initial search. After the screening of titles and abstracts, 603 potentially eligible studies were retrieved for further analysis among which, 579 RCTs were excluded from further analysis according to reviewing the full text of the studies because they were out of the borderline of expected outcomes. Finally, 18 RCTs entered to meta-analysis. - Fig. 1 shows a detailed explanation regarding the processes of study selection step by step. - Table 1 presents the key characteristics of the RCTs. The eighteen selected studies enrolled 2009 


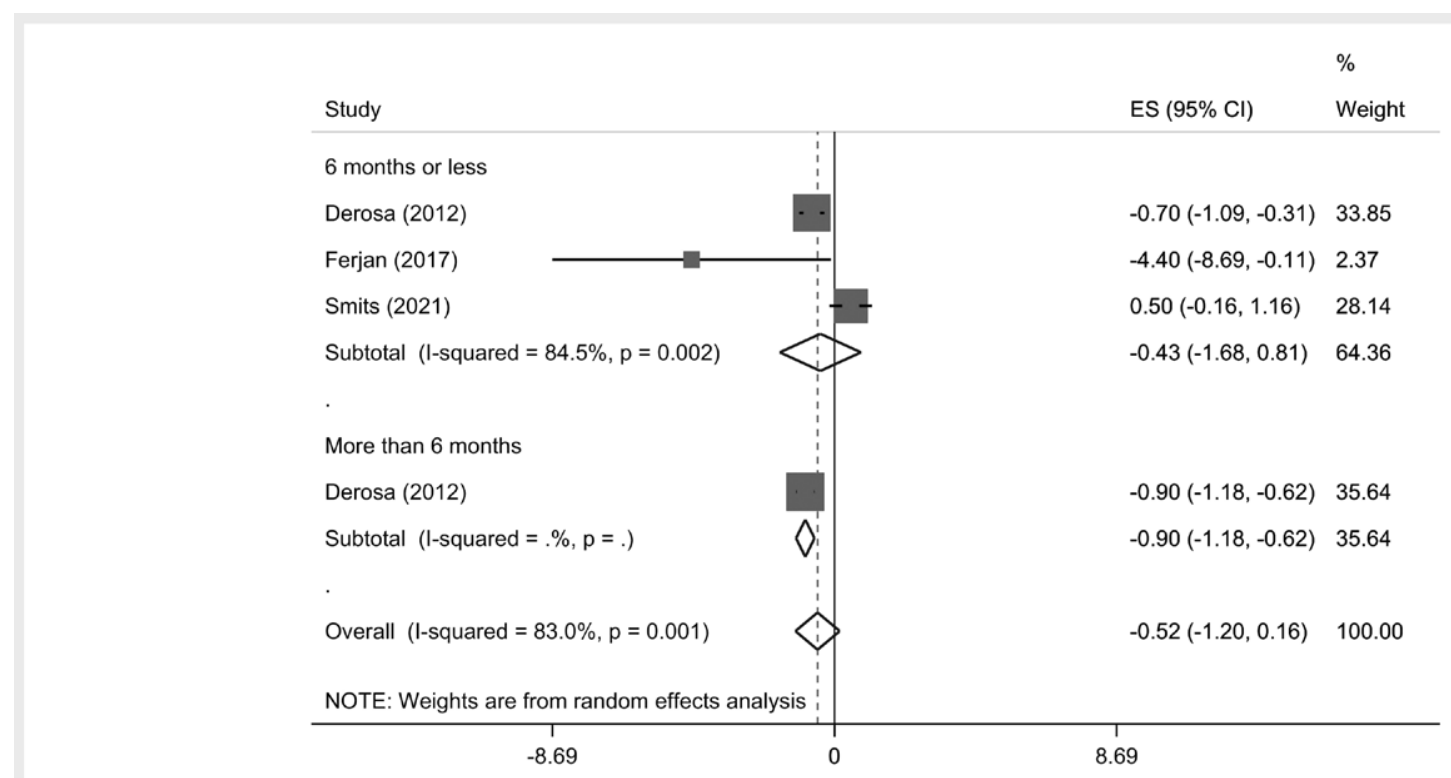

- Fig. 10 Point estimation of mean differences with $95 \% \mathrm{Cl}$ for BMI in multiple drug groups for comparison of sitagliptin group vs. placebo. Forest plot using random-effect-model meta-analysis for comparison of BMI means in people who received sitagliptin + metformin vs. people who received placebo (ES; effect size, Weight: study weight in meta-analysis).

- Table 2 Subgroup analysis of the effects of sitagliptin supplementation as monotherapy and add on to metformin ('single drug' means sitagliptin, and 'multidrug' mean sitagliptin + metformin).

\begin{tabular}{|c|c|c|c|c|c|}
\hline Variable & Intervention type & No. & Subgroups & Pooled effect estimate & $95 \% \mathrm{Cl}$ \\
\hline \multirow[t]{4}{*}{ Weight (Kg) } & \multirow{2}{*}{$\begin{array}{l}\text { Intervention group (after vs. } \\
\text { before) }\end{array}$} & 5 & Single-drug & -0.99 & $(-1.87,-0.12)$ \\
\hline & & 7 & Multidrug & -1.09 & $(-1.69,-0.49)$ \\
\hline & \multirow{2}{*}{$\begin{array}{l}\text { Change intervention group vs. } \\
\text { placebo group }\end{array}$} & 3 & Single-drug & 0.959 & $(-3.398,5.317)$ \\
\hline & & 7 & Multidrug & 0.13 & $(-0.90,1.17)$ \\
\hline \multirow[t]{4}{*}{ BMI } & \multirow{2}{*}{$\begin{array}{l}\text { Intervention group (after vs. } \\
\text { before) }\end{array}$} & 4 & Single drug & -0.23 & $(-0.45,-0.02)$ \\
\hline & & 6 & Multidrug & -0.520 & $(-0.96,-0.08)$ \\
\hline & \multirow{2}{*}{$\begin{array}{l}\text { Change intervention group vs. } \\
\text { the placebo group }\end{array}$} & 1 & Single-drug & -0.533 & $(-1.281,0.216)$ \\
\hline & & 2 & Multidrug & -0.52 & $(-1.199,0.16)$ \\
\hline
\end{tabular}

participants. All the included studies had been published after 2010. The participants in these trials were aged between 18 to 70 years old. Among all included studies, ten trials reported changes in BMI and weight [28, 29, 35-42], two studies only reported BMI $[43,44]$, and six studies only reported body weight [45-50].

\section{Effect of Sitagliptin on Body Weight}

Generally, seven selected RCTs reported the effects of supplementation of sitagliptin on body weight. Meta-analysis of the data indicated that, supplementation of sitagliptin has not led to body weight reduction in single-drug group for 6 months or less (MD -0.54; $95 \% \mathrm{Cl} ;(-1.11,0.04) ; \mathrm{p}=0.066)$ ), but the results showed it had significant effect for more than 6 months (MD -1.68; $95 \% \mathrm{Cl}$; (-3.19, -0.16); $\mathrm{p}=0.030)$ - Fig. 3).

Further analysis before and after the intervention indicated the same pattern for multiple-drug groups. supplementation of the sitagliptin did not influence bodyweight reduction in the multiple-drug group for 6 months or less (MD -0.58 ; $95 \% \mathrm{Cl}$; $(-1.23,0.07)$; $p=0.081)$ ), but it had significant effect for more than 6 months (MD -1.48; 95\% Cl; (-2.69, -0.27); $\mathrm{p}=0.016)$ ) as shown in > Fig. 4.

Also, results of comparison between intervention and control groups revealed that, treatment with sitagliptin as monotherapy had not a significant effect on reduction of body weight during 6 months or less (MD 2.70; $95 \% \mathrm{Cl} ;(-4.03,9.43] ; \mathrm{p}=0.431)$ ) and more than 6 months (MD $-1.60 ; 95 \% \mathrm{Cl} ;(-8.17,4.97) ; \mathrm{p}=0.633)$ ) in single -drug group as shown in - Fig. 5 and (MD $-058 ; 95 \% \mathrm{Cl}$; $(-0.83,1.98) ; \mathrm{p}=0.422)$ ) during 6 months or less and (MD -0.40 ; $95 \% \mathrm{Cl} ;(-1.94,1.14) ; \mathrm{p}=0.610))$ for more than 6 months as multiple -drug group, respectively ( $>$ Fig. 6).

\section{Effect of Sitagliptin on BMI}

Although the supplementation of sitagliptin had not influenced BMI through separate subgroups of time ( $P$-value $>0.05$ ), the results of the meta-analysis showed a significant difference in overall time points ( $\mathrm{MD}-0.23 ; 95 \% \mathrm{Cl} ;(-0.45,-0.02] ; \mathrm{p}=0.033)$ ) in the monotherapy group ( $\triangleright$ Fig. 7 ). Besides, the sitagliptin as an addon to metformin has not influenced the BMI in multiple -drug group 
a

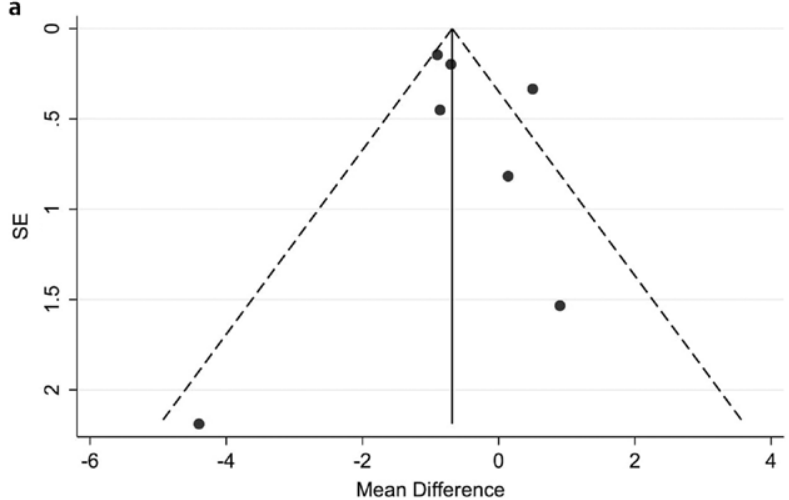

c

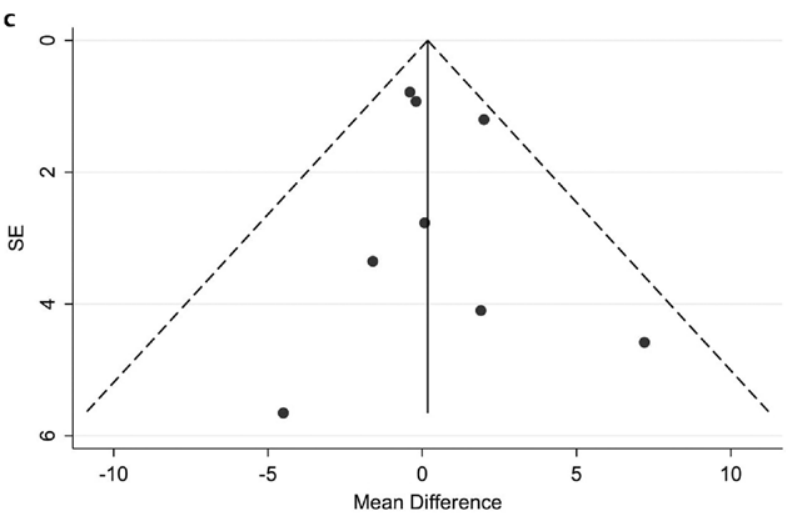

b

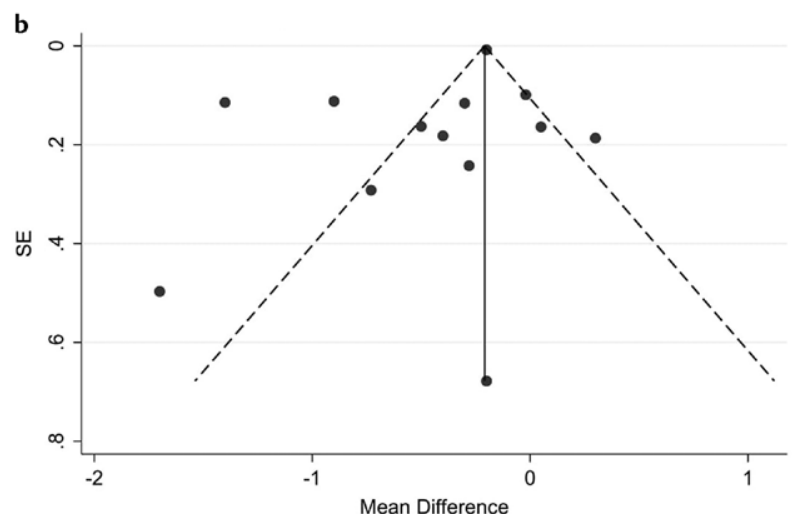

d

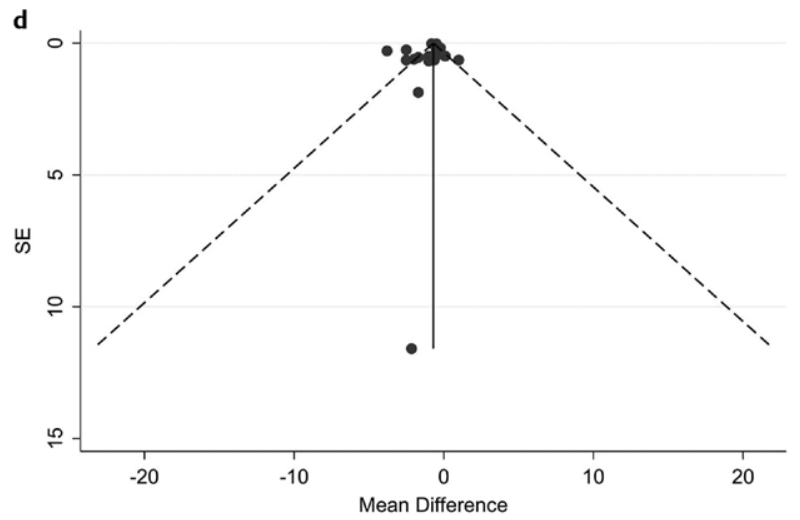

- Fig. 11 a. Funnel plot for BMI comparison of intervention group vs. placebo. Funnel plot with $95 \%$ confidence limit for BMI comparison of intervention group vs. placebo (SE: standard error) b. Funnel plot for before-after comparison of BMI in the intervention group. Funnel plot with $95 \%$ confidence limit for before-after comparison of BMI in the intervention group (SE: standard error) c. Funnel plot for body weight for comparison of sitagliptin group vs. placebo. Funnel plot with $95 \%$ confidence limit for body weight for comparison of sitagliptin group vs. placebo (SE: standard error) d. Funnel plot for before-after comparison of body weight in intervention group. Funnel plot with $95 \%$ confidence limit for before-after comparison of body weight in the intervention group (SE: standard error).

during 6 months (MD $-0.15 ; 95 \% \mathrm{Cl} ;(-0.53,0.24) ; \mathrm{p}=0.462)$ ) but showed a significant effect in more than 6 months, (MD $-0.88 ; 95 \%$ $\mathrm{Cl} ;(-1.34,-0.42) ; \mathrm{p}<0.001)$ ) ( Fig. 8).

Additionally, the effect of sitagliptin administration on BMI and Weight was analyzed between intervention and control groups. A significant reduction has not been shown in the pooled MDs for sitagliptin as monotherapy or with metformin compared to placebo. Mean differences of intervention-control comparison were (MD - 0.53; $95 \% \mathrm{Cl} ;(-1.28,0.22) ; \mathrm{p}=0.163)$ ) and (MD $-0.52 ; 95 \%$ $\mathrm{Cl} ;(-1.20,0.62) ; \mathrm{p}=0.134))$ respectively., as depicted in detail in

\section{Fig. 9 and $>$ Fig. 10.}

- Table 2 illustrates the effects of the intake of sitagliptin on body weight and BMI based on subgroup analysis. Findings of stratified analyses revealed that the effects were different due to the type of intervention.

\section{Publication Bias Assessment}

Evidence of publication bias was visually detected using funnel plot asymmetry and quantified by Egger's test $(P<0.10)$. Visual inspection of the funnel plot represented no asymmetry, and there were no small-study effects or publication biases as assessed by Egger's test ( Fig. 11a-d).

\section{Risk of Bias Assessment for RCTs}

The quality evaluation of the included studies was based on Cochrane Handbook for Systematic Reviews of Interventions, and - Fig. 2a, b present the results obtained from the risk of bias assessment.

\section{Discussion}

Considerable attention has been devoted to weight loss in recent years. It has been well established that obesity is associated with an increased risk of developing diabetes and other complications such as insulin resistance and cardiovascular diseases [51-54]. Previous studies acknowledged the critical role of obesity, and it has been reported that more than two-thirds of the individuals who have type 2 diabetes are overweight or obese [55]. It is well known that weight loss leads to significant improvements in insulin secretion, sensitivity, reduction in cardiovascular risk, and better outcomes for patients with type 2 diabetes[56]. Therefore, weight loss is a significant area of interest during the treatment of obese subjects through glycemic control. DPP-4 inhibitors are known as a novel class of glucose-lowering agents, which have been reported to reduce blood glucose levels by inhibiting DPP-4 [57] and improving pancreatic $\beta$-cell function in type 2 diabetes. The 
sitagliptin is classified as one of the first DPP-4 inhibitors approved by the FDA for clinical use in 2006 [58]. Now, two inhibitors include vildagliptin and sitagliptin, are available on the market for oral administration. The sitagliptin can be used in combination with other antidiabetic drugs such as metformin, sulfonylurea, or thiazolidinedione compounds for individuals with inadequate glycemic control after treatment with traditional antidiabetic medications.

Though substantial research has been conducted on the influence of DPP-4 inhibitors on reducing weight and BMI, there are somewhat controversial experimental data about the effect of sitagliptin. There is no general agreement on the fact that sitagliptin has the potential to reduce weight [59].

Previous studies only focused on all three DPP-4 inhibitors, and they have been reported not to affect weight $[60,61]$. In this regard, no comprehensive systematic review has been conducted to determine the effect of sitagliptin on body weight. So, we can say that a meta-analysis of RCTs provides a comprehensive assessment to gain a detailed understanding of the impact of sitagliptin on weight loss. However, further prospective studies are needed to investigate the weight loss effect of this drug to develop a complete picture regarding the usage of sitagliptin.

The lack of enough information on available trials was a limitation in the present study. For instance, in several trials, the sitagliptin's evaluation of the weight loss effect had not been reported as a preferred outcome at the end of the treatment. Despite the use of random effect models and subgroup analysis, which mainly led to the reduction of $\mathrm{I}^{2}$ statistic of heterogeneity, lack of large sample studies, and presence of heterogeneous studies is one of the considerable limitations of this study.

\section{Conclusions}

This review identified that administration of sitagliptin solely and in combination with metformin for more than 6 months has improved body weight and body mass index reduction.

Further research studies are required to explore this association. This may subsequently help to improve interventions for weight management of people with type 2 diabetes.

\section{Acknowledgments}

This study was supported by grant No. 12978 from Iran University of Medical Sciences.

\section{Competing Interests}

The authors declare that they have no competing interests.

\section{References}

[1] Nguyen T, Lau DC. The obesity epidemic and its impact on hypertension. Canadian Journal of Cardiology 2012; 28 (3): 326-333

[2] Popa AR, Fratila O, Rus M et al. Risk factors for adiposity in the urban population and influence on the prevalence of overweight and obesity. Experimental and Therapeutic Medicine 2020; 20: 129-133
[3] Grundy SM. Multifactorial causation of obesity: implications for prevention. The American Journal of Clinical Nutrition 1998; 67: 563S-572sS

[4] Wang YC, McPherson K, Marsh T et al. Health and economic burden of the projected obesity trends in the USA and the UK. The Lancet 2011; 378: $815-825$

[5] Guo X, Zhang T, Shi L et al. The relationship between lipid phytochemicals, obesity and its related chronic diseases. Food \& Function 2018; 9 : 6048-6062

[6] Anderson JW, Kendall CW, Jenkins DJ. Importance of weight management in type 2 diabetes: review with meta-analysis of clinical studies. Journal of the American college of nutrition 2003; 22: 331-339

[7] Wing RR, Lang W, Wadden TA et al. Benefits of modest weight loss in improving cardiovascular risk factors in overweight and obese individuals with type 2 diabetes. Diabetes care 2011; 34: 1481-1486

[8] O’Keefe Jr JH, Miles JM, Harris WH et al.editors. Improving the adverse cardiovascular prognosis of type 2 diabetes. Mayo Clinic Proceedings 1999; 74(2):171-180

[9] Duckworth WC, McCarren M, Abraira C. Glucose control and cardiovascular complications: the VA Diabetes Trial. Diabetes Care 2001; 24: 942-945

[10] Amatruda JM, Richeson JF, Welle SL et al. The safety and efficacy of a controlled low-energy ('very-low-calorie') diet in the treatment of non-insulin-dependent diabetes and obesity. Archives of Internal Medicine 1988; 148: 873-877

[11] Stern MP, Patterson JK, Haffner SM et al. Lack of awareness and treatment of hyperlipidemia in type II diabetes in a community survey. JAMA 1989; 262: 360-364

[12] Velazquez A, Apovian CM. Updates on obesity pharmacotherapy. Annals of the New York Academy of Sciences 2018; 1411: 106-119

[13] Association AD. Standards of Medical Care in Diabetes-2021 Abridged for Primary Care Providers. Clinical Diabetes 2021; 39: 14-43

[14] Phung OJ, Scholle JM, Talwar M et al. Effect of noninsulin antidiabetic drugs added to metformin therapy on glycemic control, weight gain, and hypoglycemia in type 2 diabetes. Jama 2010; 303: 1410-1418

[15] Robinson LE, Holt TA, Rees K et al. Effects of exenatide and liraglutide on heart rate, blood pressure and body weight: systematic review and meta-analysis. BMJ open 2013; 3(1): e001986

[16] Derosa G, Maffioli P. Dipeptidyl peptidase- 4 inhibitors: 3 years of experience. Diabetes technology \& therapeutics 2012; 14: 350-364

[17] Butryn ML, Webb V, Wadden TA. Behavioral treatment of obesity. Psychiatric Clinics 2011; 34: 841-859

[18] Drucker D, Easley C. Kirkpatrick P. Sitagliptin. Nature Reviews Drug Discovery 2007; 6: 109

[19] Holst J]. The physiology of glucagon-like peptide 1. Physiological reviews 2007; 87: 1409-1439

[20] Holst ]J, Vilsbøll T, Deacon CF. The incretin system and its role in type 2 diabetes mellitus. Molecular and cellular endocrinology 2009; 297: 127-136

[21] Drucker D]. The biology of incretin hormones. Cell Metabolism 2006; 3: 153-165

[22] Schirra J, Göke B. The physiological role of GLP-1 in human: incretin, ileal brake or more? Regulatory Peptides 2005; 128: 109-115

[23] Aulinger BA, Bedorf A, Kutscherauer $G$ et al. Defining the role of GLP-1 in the enteroinsulinar axis in type 2 diabetes using DPP-4 inhibition and GLP-1 receptor blockade. Diabetes 2014; 63: 1079-1092

[24] Scott L]. Sitagliptin: a review in type 2 diabetes. Drugs. 2017; 77: 209-224

[25] Ferreira L, Teixeira-de-Lemos E, Pinto F et al. Effects of sitagliptin treatment on dysmetabolism, inflammation, and oxidative stress in an animal model of type 2 diabetes (ZDF rat). Mediators of inflammation 2010; 2010

[26] Tran S, Retnakaran R, Zinman B et al. Efficacy of glucagon-like peptide-1 receptor agonists compared to dipeptidyl peptidase- 4 inhibitors for the management of type 2 diabetes: A meta-analysis of randomized clinical trials. Diabetes, Obesity and Metabolism 2018; 20: 68-76 
[27] Ling J, Cheng P, Ge L et al. The efficacy and safety of dipeptidyl peptidase-4 inhibitors for type 2 diabetes: a Bayesian network meta-analysis of 58 randomized controlled trials. Acta Diabetologica 2019; 56: 249-272

[28] Garg SK, Moser EG, Bode BW et al. Effect of sitagliptin on post-prandial glucagon and GLP-1 levels in patients with type 1 diabetes: investigator-initiated, double-blind, randomized, placebo-controlled trial. Endocrine Practice 2013; 19: 19-28

[29] Gül ÖÖ, Kıyıc S, Ersoy C et al. Effect of sitagliptin monotherapy on serum total ghrelin levels in people with type 2 diabetes. Diabetes research and clinical Practice 2011; 94: 212-216

[30] Lavalle-González F, Januszewicz A, Davidson J et al. Efficacy and safety of canagliflozin compared with placebo and sitagliptin in patients with type 2 diabetes on background metformin monotherapy: a randomised trial. Diabetologia. 2013; 56: 2582-2592

[31] Ristic S, Byiers S, Foley J et al. Improved glycaemic control with dipeptidyl peptidase- 4 inhibition in patients with type 2 diabetes: vildagliptin (LAF237) dose response. Diabetes. Obesity and Metabolism 2005; 7: 692-698

[32] Pi-Sunyer FX, Schweizer A, Mills D et al. Efficacy and tolerability of vildagliptin monotherapy in drug-naive patients with type 2 diabetes. Diabetes Research and Clinical Practice 2007; 76: 132-138

[33] Higgins ], Julian PT, Altman DG. Assessing risk of bias in included studies. Cochrane Handbook for Systematic Reviews of Interventions: Cochrane Book Series 2008; 187-241

[34] Borenstein M, Higgins ], Hedges LV et al. Basics of meta-analysis: $\mathrm{I}^{2}$ is not an absolute measure of heterogeneity in a meta-analysis. Res Synth Methods. 2017;8(1):5-18.

[35] Fuchigami A, Shigiyama F, Kitazawa T et al. Efficacy of dapagliflozin versus sitagliptin on cardiometabolic risk factors in Japanese patients with type 2 diabetes: a prospective, randomized study (DIVERSITYCVR). Cardiovascular diabetology 2020; 19: 1-11

[36] Webb DR, Htike ZZ, Swarbrick D] et al. A randomized, open-label, active comparator trial assessing the effects of 26 weeks of liraglutide or sitagliptin on cardiovascular function in young obese adults with type 2 diabetes. Diabetes, Obesity and Metabolism 2020; 22: 1187-1196

[37] Derosa G, Carbone A, Franzetti I et al. Effects of a combination of sitagliptin plus metformin vs metformin monotherapy on glycemic control, $\beta$-cell function and insulin resistance in type 2 diabetic patients. Diabetes Research and Clinical Practice 2012; 98: 51-60

[38] Ferjan S, Janez A, Jensterle M. Dipeptidyl peptidase-4 inhibitor sitagliptin prevented weight regain in obese women with polycystic ovary syndrome previously treated with liraglutide: a pilot randomized study. Metabolic syndrome and related disorders 2017; 15: 515-520

[39] Smits MM, Fluitman KS, Herrema $\mathrm{H}$ et al. Liraglutide and sitagliptin have no effect on intestinal microbiota composition: A 12-week randomized placebo-controlled trial in adults with type 2 diabetes. Diabetes \& Metabolism 2021; 101223

[40] Yan ], Yao B, Kuang $\mathrm{H}$ et al. Liraglutide, sitagliptin, and insulin glargine added to metformin: the effect on body weight and intrahepatic lipid in patients with type 2 diabetes mellitus and nonalcoholic fatty liver disease. Hepatology. 2019; 69: 2414-2426

[41] Kitazawa M, Katagiri T, Suzuki H et al. A 52-week randomized controlled trial of ipragliflozin or sitagliptin in type 2 diabetes combined with metformin: The N-ISM study. Diabetes, Obesity and Metabolism 2021; 23: 811-821

[42] Ji L, Dong X, Li Y et al. Efficacy and safety of once-weekly semaglutide vs once-daily sitagliptin as add-on to metformin in patients with type 2 diabetes (SUSTAIN China): a 30-week double-blind, phase 3a, randomised trial. Diabetes, Obesity and Metabolism 2021; 23:404-414

[43] Bhosle DS, Bhagat AH, Patil AD et al. Effects of a fixed-dose combination of sitagliptin and metformin versus respective monotherapies in newly diagnosed type 2 diabetic subjects. International Journal of Diabetes in Developing Countries 2017; 37: 16-20
[44] Hussain M, Rafique MA, Iqbal J et al. Effect of sitagliptin and glimepiride on C-reactive protein (CRP) in overweight Type-2 diabetic patients. Pakistan Journal of Medical Sciences 2019; 35: 383

[45] Ajmani AK, Agrawal A, Prasad B et al. Efficacy and safety of evogliptin versus sitagliptin as an add-on therapy in Indian patients with type 2 diabetes mellitus inadequately controlled with metformin: A 24-week randomized, double-blind, non-inferiority, EVOLUTION INDIA study. Diabetes Research and Clinical practice 2019; 157: 107860

[46] Halvorsen YD, Lock JP, Zhou W et al. A 24-week, randomized, double-blind, active-controlled clinical trial comparing bexagliflozin with sitagliptin as an adjunct to metformin for the treatment of type 2 diabetes in adults. Diabetes, Obesity and Metabolism 2019; 21: 2248-2256

[47] Hiruma S, Shigiyama F, Hisatake $S$ et al. A prospective randomized study comparing effects of empagliflozin to sitagliptin on cardiac fat accumulation, cardiac function, and cardiac metabolism in patients with early-stage type 2 diabetes: the ASSET study. Cardiovascular Diabetology 2021; 20: 1-13

[48] Gadde KM, Vetter ML, lqbal N et al. Efficacy and safety of autoinjected exenatide once-weekly suspension versus sitagliptin or placebo with metformin in patients with type 2 diabetes: T he DURATION-NEO-2 randomized clinical study. Diabetes, Obesity and Metabolism 2017; 19: $979-988$

[49] Aaboe K, Knop F, Vilsbøll T et al. Twelve weeks treatment with the DPP-4 inhibitor, sitagliptin, prevents degradation of peptide YY and improves glucose and non-glucose induced insulin secretion in patients with type 2 diabetes mellitus. Diabetes, Obesity and Metabolism 2010; 12: 323-333

[50] Rosenstock ], Allison D, Birkenfeld AL et al. Effect of additional oral semaglutide vs sitagliptin on glycated hemoglobin in adults with type 2 diabetes uncontrolled with metformin alone or with sulfonylurea: the PIONEER 3 randomized clinical trial. Jama. 2019; 321: 1466-1480

[51] Strasser B, Schobersberger W. Evidence for resistance training as a treatment therapy in obesity. Journal of Obesity 2011; 2011

[52] Sallam RM, Alayoubi SMZ, Al-Daghri NM et al. Gender-Specific profiles of cardiovascular disease in type 2 diabetes mellitus: A cross-sectional study. Journal of Nature and Science of Medicine 2018; 1: 74

[53] Kopelman P. Health risks associated with overweight and obesity. Obesity reviews 2007; 8: 13-17

[54] Fernandes JC, Collaborators GO. Health effects of overweight and obesity in 195 countries over 25 years. New England Journal of Medicine 2017; 377: 13-27

[55] Shin SJ. Glucagon-like peptide-1 receptor agonists and their effects on weight reduction. Journal of Diabetes Investigation 2012; 3: 490

[56] Pareek M, Schauer PR, Kaplan LM et al. Metabolic surgery: weight loss, diabetes, and beyond. Journal of the American College of Cardiology 2018; 71: 670-687

[57] Nauck M, Meininger G, Sheng Do et al. Efficacy and safety of the dipeptidyl peptidase-4 inhibitor, sitagliptin, compared with the sulfonylurea, glipizide, in patients with type 2 diabetes inadequately controlled on metformin alone: a randomized, double-blind, non-inferiority trial. Diabetes, Obesity and Metabolism 2007; 9: 194-205

[58] Dicker D. DPP-4 inhibitors: impact on glycemic control and cardiovascular risk factors. Diabetes care 2011; 34: S276-S278

[59] Lovshin JA, Drucker DJ. Incretin-based therapies for type 2 diabetes mellitus. Nature Reviews Endocrinology 2009; 5: 262

[60] Amori RE, Lau J, Pittas AG. Efficacy and safety of incretin therapy in type 2 diabetes: systematic review and meta-analysis. Jama. 2007; 298: 194-206

[61] Sethi MK, Singh D, Murti K et al. DPP-4 inhibitors: A novel approach for management of type-2 diabetes mellitusWorld Journal of Pharmaceutical Sciences 2014; 2(10); 